\title{
Self-Cholesterol Measuring Devices Mission and CardioChek: Total Cholesterol, Triglycerides, High Density Lipoprotein (HDL)-Cholesterol Estimations in Whole Blood Samples
}

\author{
S Clark ${ }^{1}$ and J Bolodeoku ${ }^{2 *}$ \\ ${ }^{1} J B$ Consulting MDP Limited, 1 Bell Street, Maidenhead, UK \\ ${ }^{2}$ Department of Cardiology, Lipid Clinic, North Hampshire Hospital Foundation Trust, UK
}

*Corresponding author: J Bolodeoku, Department of Cardiology, Lipid Clinic, North Hampshire Hospital Foundation Trust, Basingstoke, Hampshire, UK

\section{ARTICLE INFO}

Received: May 03, 2021

Published: 蔧 May 10, 2021

Citation: S Clark, J Bolodeoku. SelfCholesterol Measuring Devices Mission and CardioChek: Total Cholesterol, Triglycerides, High Density Lipoprotein (HDL)-Cholesterol Estimations in Whole Blood Samples. Biomed J Sci \& Tech Res 35(4)-2021. BJSTR. MS.ID.005735.

Abbreviations: TC: Total Cholesterol; TG: Triglycerides; HDL-C: High Density Lipoprotein-Cholesterol; LDL-C: Low Density Lipoprotein-Cholesterol; NCEP: National Cholesterol Education Program

\section{ABSTRACT}

Self-monitoring of blood lipids has been described as very important in the management of the patients with raised blood lipids. There are a number of selfmonitoring blood lipid devices such as the Mission ACON and the CardioChek PA. They measure a number of lipid fractions: total cholesterol (TC), triglycerides (TG), high density lipoprotein-cholesterol (HDL-C), low density lipoprotein-cholesterol (LDL-C) and ratios on whole blood, plasma or serum. Both devices have been shown to meet most of the criteria of analytical acceptability by the National Cholesterol Education Program (NCEP) guidelines. We compared the performance of the Mission and CardioChek lipid testing devices to determine lipid profile (total cholesterol, HDL-cholesterol and triglycerides) in whole blood samples from healthy volunteers. The total cholesterol estimates of both devices showed good correlation ( $\mathrm{r}=0.83)$, with the Mission's estimates being significantly higher than CardioChek estimates, on average by $1.29 \mathrm{mmol} / \mathrm{L}$ (22.4\%). The HDL-cholesterol estimates of both devices showed good correlation ( $\mathrm{r}=0.87$ ), with the Mission's estimates being significantly higher than the CardioChek estimates, on average by $0.16 \mathrm{mmol} / \mathrm{L}(7.6 \%)$. The triglyceride estimates of both devices showed very good correlation ( $r=0.99$ ), with the Mission's estimates being slightly lower than the CardioChek estimates, on average by $0.08 \mathrm{mmol} / \mathrm{L}(15.9 \%)$.

\section{Introduction}

Hyperlipidemia is considered a primary and independent risk factor for cardiovascular disease. The monitoring of the blood lipids has been considered very important in the management of the disease. Self-monitoring of blood lipids has been described as very important in the management of the patients with raised blood lipids. There are a number of self-monitoring blood lipid devices such as CardioChek PA and Mission ACON. They measure a number of lipid fractions (total cholesterol, triglycerides, high density lipoprotein, low density lipoprotein) and ratios on whole blood, plasma or serum collected from the finger or venous blood using reflectance or biosensor technology with single-use, disposable, dry reagent test strips, rotors or cassettes. The Mission ACON has recently been described to meet most of the criteria (precision, accuracy and total analytical error) of analytical acceptability by the NCEP guidelines [1]. The CardioChek has been described to show reasonable compliance with NCEP guidelines for coefficient of variation and bias measurements [2,3]. It's measurements (total cholesterol, HDL - cholesterol, triglycerides and LDL - cholesterol) have shown good clinical agreement and correlation with reference laboratory analysers $[4,5]$.

We have previously shown that total cholesterol estimations have been lower on the CardioChek device compared with the 
estimations made using the Mission device [6]. In addition, there has been a report which compared the cholesterol estimations from Mission and CardioChek devices to a reference method, the cholesterol estimations from both devices showed similar agreement to the reference method [7]. In this short report, we have compared both the performance of the Mission ACON and CardioChek PA in measuring lipid profile (total cholesterol, HDLcholesterol and triglycerides) in whole blood samples from healthy volunteers.

\section{Material and Methods}

\section{Materials}

Whole blood samples $(n=25)$ were collected from healthy blood donor volunteers (15 females and 10 males) according to IRB conditions by Cambridge Biosciences, UK. These blood sample collections are carried out in partnership with London-based Research Donors. Research Donors is a HTA licensed and ISO 9001 2015 certified company with Research Ethics approval (REC) as a tissue bank. The blood samples were collected into lithium heparin tubes.

Ethical Statement: Consent was provided by the subject to use the data provided. All samples were collected under a comprehensive informed consent that explicitly permits the use of blood samples and derivatives in a wide range of research applications, including commercial research. Samples are anonymised but traceable to origin.

\section{Methods}

a) Mission-Total cholesterol (TC), HDL-cholesterol and triglyceride measurements were carried out according to the manufacturer's instruction.

b) CardioChek PA-Total cholesterol, HDL-cholesterol and triglyceride measurements were carried out according to the manufacturer's instruction.

Statistical Analysis: All analyses were performed by GraphPad Prism 9.0.2: descriptive statistics [minimum, maximum, mean and standard deviation (SD)]. Pearson correlation, Bland-Altman (bias and agreement) and Paired $\mathrm{T}$ tests with estimation plots were performed.

\section{Results}

\section{Descriptive Analysis}

The Mission's total cholesterol (TC) estimates ranged between 3.6 and $9.8 \mathrm{mmol} / \mathrm{L}$, mean $5.6 \mathrm{mmol} / \mathrm{L}$ and $\mathrm{SD}+/-1.5 \mathrm{mmol} / \mathrm{L}$ compared to the CardioChek's total cholesterol of 2.6 and 6.9 mmol/L, mean $4.3 \mathrm{mmol} / \mathrm{L}$ and SD +/-1.1 mmol/L (Table 1). The Mission's HDL- cholesterol estimates ranged between 0.9 and 2.6 $\mathrm{mmol} / \mathrm{L}$, mean $2.0 \mathrm{mmol} / \mathrm{L}$ and SD $0.6 \mathrm{mmol} / \mathrm{L}$ compared to the CardioChek's total cholesterol of 1.0 and $3.0 \mathrm{mmol} / \mathrm{L}$, mean 1.9 $\mathrm{mmol} / \mathrm{L}$ and SD $0.6 \mathrm{mmol} / \mathrm{L}$. The Mission's triglyceride estimates ranged between 0.5 and $6.1 \mathrm{mmol} / \mathrm{L}$, mean $1.2 \mathrm{mmol} / \mathrm{L}$ and SD 1.1 $\mathrm{mmol} / \mathrm{L}$ compared to the CardioChek's total cholesterol range of 0.7 and $5.7 \mathrm{mmol} / \mathrm{L}$, mean $1.3 \mathrm{mmol} / \mathrm{L}$ and SD $1.0 \mathrm{mmol} / \mathrm{L}$.

Table 1: Showing total cholesterol, HDL-cholesterol and triglyceride measurements using the Mission and CardioChek devices in the volunteers.

\begin{tabular}{|c|c|c|c|c|c|c|}
\hline Volunteer & Mission TC & Cardiochek TC & Mission HDL-C & Cardiochek HDL-C & Mission Trigs & Cardiochek Trigs \\
\hline 1 & 6.23 & 5.22 & 2.59 & 3.01 & 0.69 & 0.8 \\
\hline 2 & 6.41 & 5.26 & 2.43 & 2.06 & 0.7 & 0.83 \\
\hline 3 & 3.85 & 2.93 & 1.27 & 1.13 & 0.69 & 0.89 \\
\hline 4 & 5.15 & 4.53 & 2.33 & 1.9 & 0.65 & 0.86 \\
\hline 5 & 6.36 & 5.62 & 2.59 & 2.83 & 1.17 & 1.29 \\
\hline 6 & 4.63 & 4.5 & 2.59 & 2.7 & 0.52 & 0.65 \\
\hline 7 & 6.34 & 4.49 & 1.19 & 1.13 & 1.02 & 1.24 \\
\hline 8 & 9.16 & 6.92 & 1.66 & 1.4 & 6.13 & 5.65 \\
\hline 9 & 4.32 & 3.24 & 2.59 & 1.92 & 0.54 & 0.69 \\
\hline 10 & 6.44 & 4.48 & 2.59 & 1.91 & 0.84 & 0.92 \\
\hline 11 & 5.02 & 3.05 & 2.38 & 2.15 & 0.72 & 0.85 \\
\hline 12 & 4.47 & 4.36 & 2.59 & 2.33 & 0.93 & 1.35 \\
\hline 13 & 4.09 & 2.91 & 2.59 & 1.82 & 0.51 & 0.71 \\
\hline 14 & 4.32 & 3.12 & 1.55 & 1.38 & 0.82 & 1.11 \\
\hline 15 & 4.99 & 3.97 & 1.76 & 1.64 & 0.58 & 0.67 \\
\hline 16 & 5.2 & 3.51 & 2.59 & 1.91 & 1.11 & 0.69 \\
\hline 17 & 6.62 & 5.06 & 1.19 & 1.13 & 0.95 & 1.14 \\
\hline 18 & 6.05 & 5.32 & 2.59 & 3.02 & 2.88 & 2.57 \\
\hline 19 & 4.16 & 2.59 & 1.53 & 1.46 & 1.39 & 1.62 \\
\hline
\end{tabular}




\begin{tabular}{|l|c|c|c|c|c|c|}
\hline 20 & 9.83 & 5.65 & 2.59 & 2.77 & 1.09 & 0.86 \\
\hline 21 & 4.71 & 3.38 & 0.91 & 0.98 & 1.67 & 0.51 \\
\hline 22 & 5.25 & 5.3 & 2.59 & 2.58 & 1.59 \\
\hline 23 & 3.57 & 2.8 & 1.35 & 1.2 & 1.72 & 1.21 \\
\hline 24 & 6.23 & 4.71 & 1.45 & 1.23 & 1.08 & 1.27 \\
\hline 25 & 6.49 & 4.73 & 1.68 & 1.59 & 1.06 & 1.32 \\
\hline
\end{tabular}

\section{Total Cholesterol}

The estimated values with the Mission were higher than the estimated values with the CardioChek and there was good correlation between the total cholesterol measurements showing a correlation coefficient $(r)$ of 0.83 . The percentage difference between the Mission and the CardioChek estimations ranged from $-0.95 \%$ (sample 22 ) to $+42.52 \%$ (sample 19), with an average percent difference of $+22.44 \%$. The Bland-Altman difference plots showed a positive bias of $1.290 \mathrm{mmol} / \mathrm{L}, \mathrm{SD}$ bias $0.8 \mathrm{mmol} / \mathrm{L}$ $(-0.3748$ to +2.954$)$. The paired $\mathrm{T}$ test indicated that there was a statistically significant difference between the two means $(\mathrm{p}<$ 0.0001).

\section{HDL-Cholesterol}

The estimated values with the Mission were higher than the estimated values with the CardioChek and there was good correlation between the total cholesterol measurements showing a correlation coefficient ( $r$ ) of 0.87 . The percentage difference between the Mission and the CardioChek estimations ranged from
$-0.16 .21 \%$ (sample 1) to $+26.25 \%$ (sample 10), the average percent difference was $+7.66 \%$. The Bland-Altman difference plots showed a positive bias of $0.1596 \mathrm{mmol} / \mathrm{L}$, with an SD bias +/- $0.3203 \mathrm{mmol} / \mathrm{L}$ $(-0.4682$ to +0.7874$)$. The paired $\mathrm{T}$ test indicated that there was a statistically significant difference between the two means $(p=0.02)$

\section{Triglyceride}

The estimated values with the Mission were lower than the estimated values with the CardioChek and there was very good correlation between the total cholesterol measurements estimated using the Mission and the CardioChek systems showing correlation coefficient ( $r$ ) of 0.99 . The percentage difference between the Mission and the CardioChek estimations ranged from $-45.16 \%$ (sample 12) to $+37.83 \%$ (sample 16), the average percent difference was $-15.59 \%$. The Bland-Altman difference plots showed a negative bias of $0.08650 \mathrm{~mol} / \mathrm{L}$, with an SD bias +/-0.2211, (-0.5189 to +0.3477 ) Figures 1-3. The paired $\mathrm{T}$ test indicated that there was no statistically significant difference between the two means $(\mathrm{p}<$ $0.0647)$.

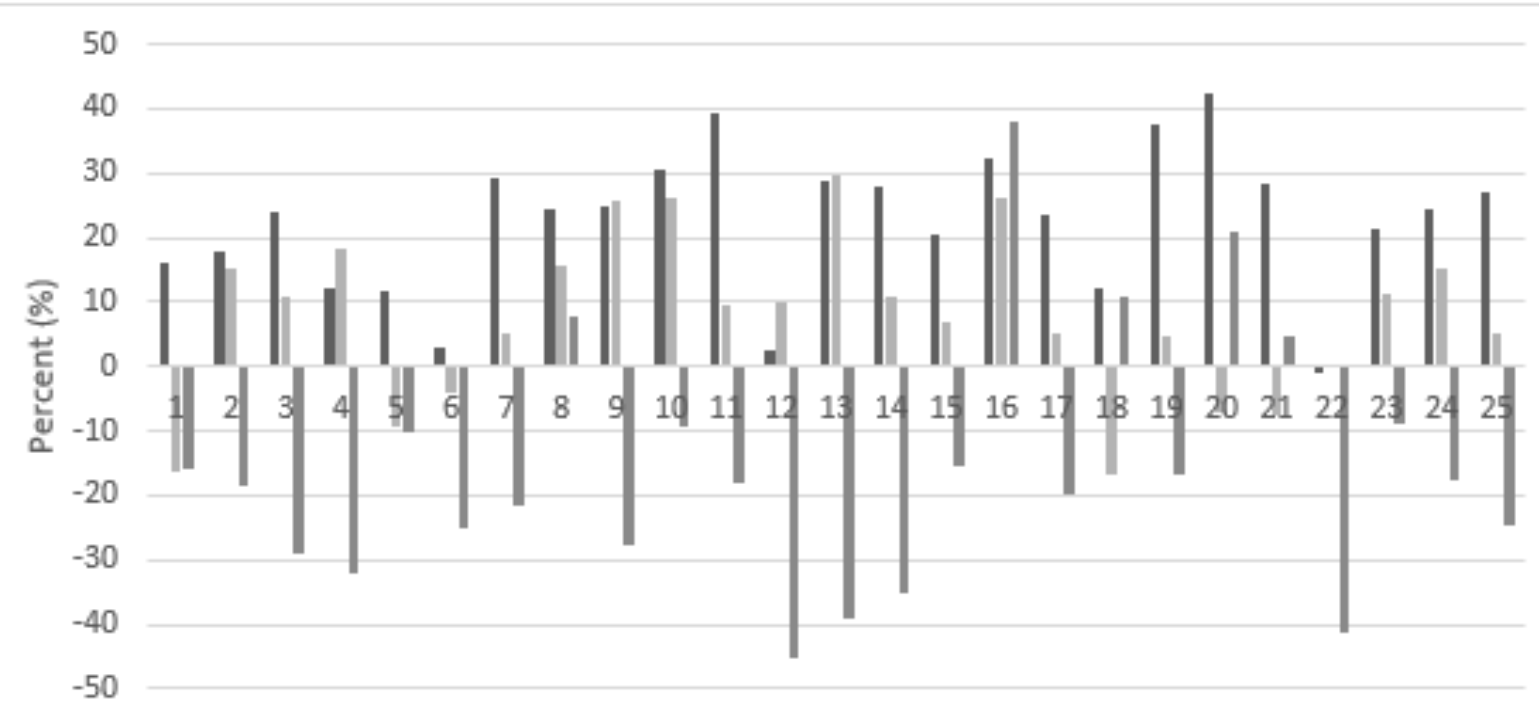

Total Cholesterol \% difference a HDL-Cholesterol \% difference a Triglyceride \% difference

Figure 1: Showing percent difference (\%) between total cholesterol, HDL-cholesterol and triglyceride estimations on the Mission and CardioChek device in whole blood samples. 

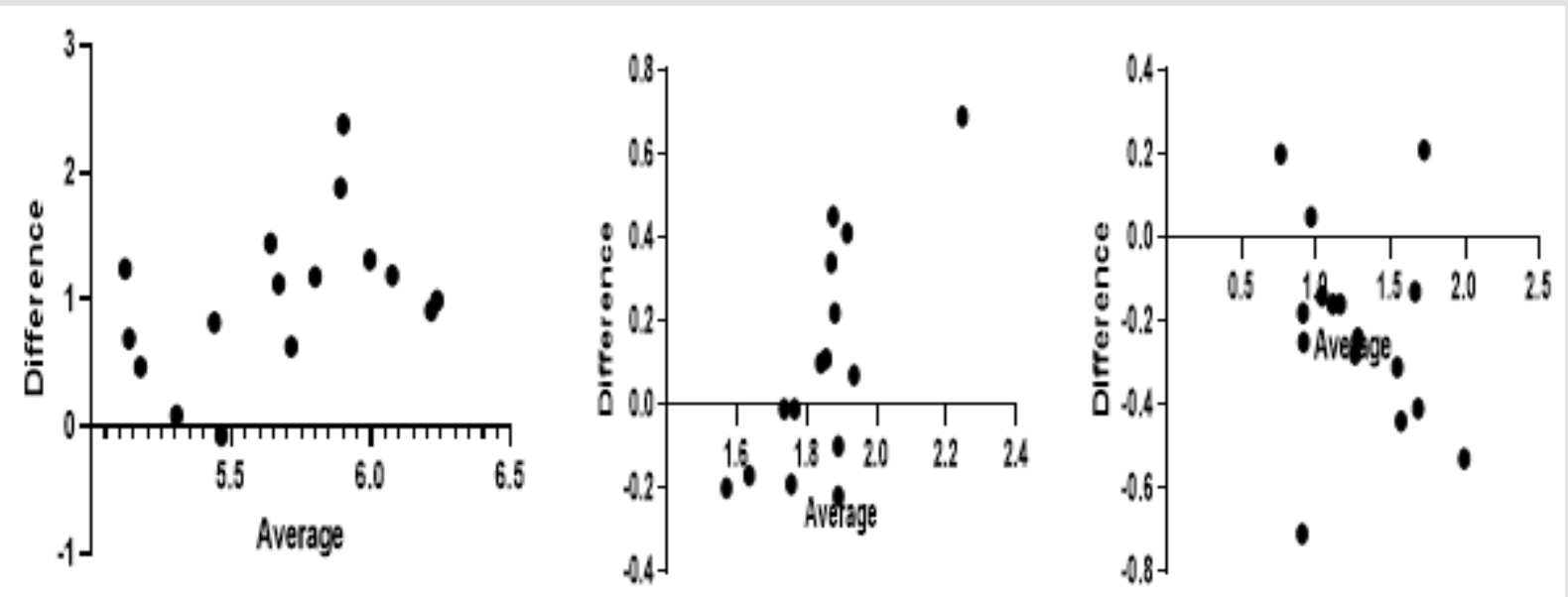

Figure 2: Bland-Altman plot comparison of the Mission and CardioChek PA's total cholesterol (left), HDL-cholesterol (middle) and triglyceride (right) measurements in paired whole blood samples. The total cholesterol, HDL-cholesterol and triglyceride data are shown on the left, middle and right panels, respectively.

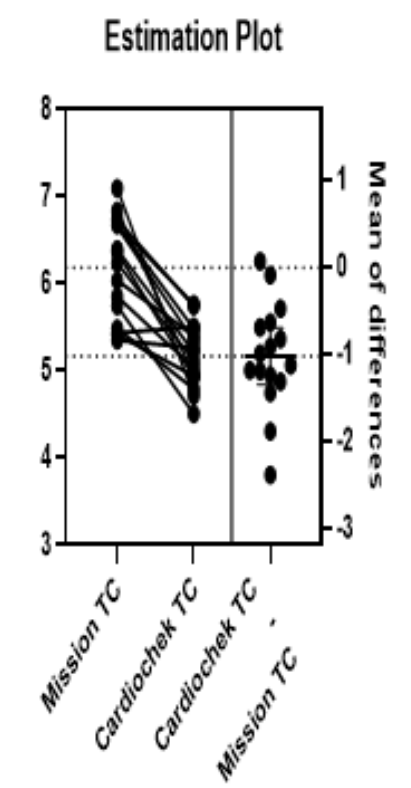

Estimation Plot

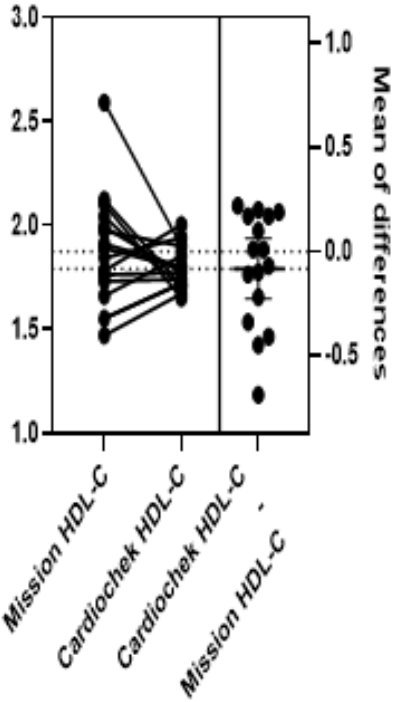

Estimation Plot

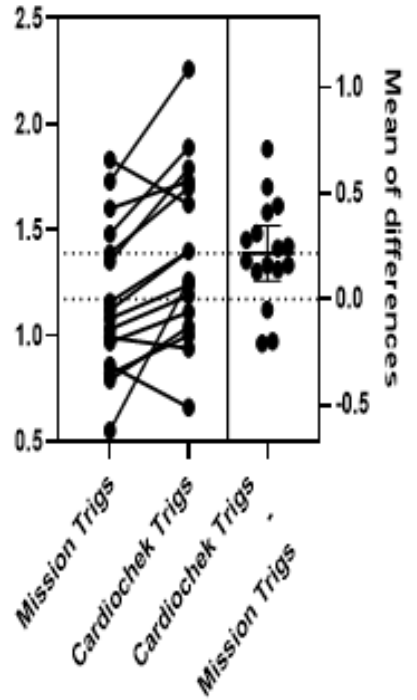

Figure 3: Estimation plots of the Mission and CardioChek PA's total cholesterol (TC), HDL-cholesterol (HDL-C) and triglyceride (trigs) measurements in paired whole blood samples. The total cholesterol, HDL- cholesterol and triglyceride data are shown on the left, middle and right panels, respectively.

\section{Discussion}

In some earlier studies, the CardioChek has been studied with other cholesterol self- monitoring devices such as the elemark, Prima 3in1, Mission 1drop. The total cholesterol estimations were observed to be lower than the other devices $[6,8,9]$. In addition, a three-year external quality control study showed that the CardioChek underestimated total cholesterol with a $6 \%$ bias [2]. In an earlier report, the Mission's total cholesterol and HDLcholesterol estimates were on average, 9.7\% and $4.6 \%$ higher than the CardioChek's total cholesterol and HDL-cholesterol estimates [7]. In this study, the Mission's total cholesterol and HDL-cholesterol estimates were on average $22.4 \%(0.8 \mathrm{mmol} / \mathrm{L})$ and average $7.6 \%$ $(0.3203 \mathrm{mmol} / \mathrm{L})$ higher than the CardioChek estimates and were statistically different. Whilst the triglyceride estimates in the earlier report using the Mission were on average 2\% lower than the CardioChek estimates. In this study, the Mission's triglycerides estimates were on average $15.59 \%(0.08650 \mathrm{~mol} / \mathrm{L})$ lower than the CardioChek estimates but not statistically significant. This 
study demonstrates and confirms the consistent lower estimations of total cholesterol and HDL-cholesterol and higher estimation of triglycerides seen using the CardioChek compared to the Mission. These differences between the cholesterol estimations of the two devices have now been confirmed in a direct head-to-head study and should be taken into consideration when these devices are being used in clinical practice especially where the differences are significant.

\section{Declaration of Conflicting Interests}

The author(s) declared no potential conflicts of interest with respect to the research, authorship, and/or publication of this article.

\section{Funding}

The author(s) received no financial support for the research, authorship, and/or publication of this article.

\section{Credit Author Statement}

S Clark (study analysis, review and editing), J Bolodeoku (writing original draft, supervision). The authors will like to thank Sarah Christie for final editing and review of the manuscript.

\section{References}

1. Quartey P, Gator D, Mpiani MA, Dapah VO (2020) Evaluation of the analytical performance of the mission cholesterol meter for serum lipids using NCEP criteria. IJMHR 6 (2): 137-139.

ISSN: 2574-1241

DOI: 10.26717/BJSTR.2021.35.005735

J Bolodeoku. Biomed J Sci \& Tech Res

CC (P) This work is licensed under Creative Commons Attribution 4.0 License

Submission Link: https://biomedres.us/submit-manuscript.php
2. Matteucci E, Bartolla LD, Rossi L, Pellegrini G, Giampietro O, et al. (2014) Improving CardioCheck PA analytical performance: a three-year study. Clin Chem Lab Med 52 (9): 1291-1296.

3. Whitehead SJ, Ford C, Gama Rousseau (2013) A combined laboratory and field evaluation of the Cholestech LDX and CardioChek PA point of care testing lipid and glucose analysers. Ann Clin Biochem 51(1): 54-67.

4. Gao Y, Zhu CG, Wu NQ, Guo YL, Liu G, et al. (2016) [Study on the reliability of CardioChek PA for measuring lipid profile]. Beijing Da Xue Xue Bao Yi Xue Ban 48(3): 523-528.

5. Ferreira CE, França CN, Correr CJ, Zucker ML, Andriolo, et al. (2015) Clinical correlation between a point-of-care testing system and laboratory automation for lipid profile. Clinica Chimica Acta 446: 263266.

6. Bolodeoku J, Clark S, Anyaeche C (2020) Self-monitoring of blood cholesterol (SMBC) using the total cholesterol testing cartridge on the 1 drop $^{\text {TM }}$ smartphone. Biomed J Sci \& Tech Res 29(2): 2020.

7. (2016) A Comparative Evaluation of Mission Cholesterol and CardioChek PA Point of Care Lipid testing systems by AZURE INSTITUTE (June 2016). Microsoft Word - A Comparative Evaluation of Mission Cholesterol and CardioChek PA POC Lipid Testing Systems-June 2016.docx.

8. Bolodeoku J, Pinkney S (2019) Imprecision evaluation of self-monitoring of blood cholesterol (SMBC) handheld point of care testing devices: Elemark $^{\mathrm{TM}}$ and CardioChek PA. Ann Clin Lab Res 7(1): 290.

9. Bolodeoku J (2018) Biological Variation of Self-Monitoring of Blood Cholesterol (SMBC) Using Portable Handheld Point of Care Testing Devices: 3in1, Cardio chek PA and Elemark. Curr Trends Med Diagn Meth 2018: CTMDM-106.

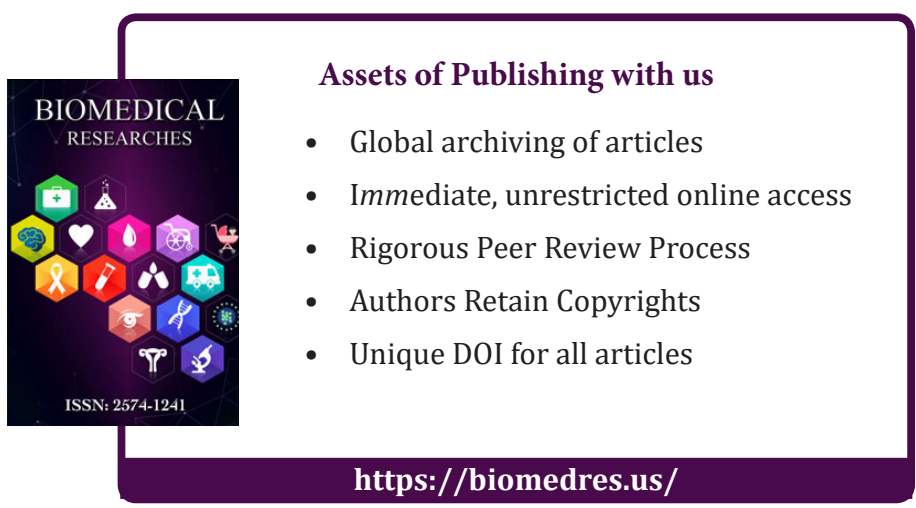

